\title{
Imaging of Wound Closure of Small Epithelial Lesions in the Mouse Trachea
}

\author{
Sarah Kretschmer, ${ }^{*}$ Mario Pieper, ${ }^{\dagger \dagger}$ Antje Klinger, ${ }^{*}$ Gereon Hüttmann, ${ }^{\dagger \dagger}$ and Peter König ${ }^{* \dagger}$
}

From the Institute of Anatomy, ${ }^{*}$ Center for Structural and Cell Biology in Medicine, and the Institute of Biomedical Optics, ${ }^{\ddagger}$ University of Lübeck, Lübeck; and the Airway Research Center North, ${ }^{\dagger}$ German Center for Lung Research (DZL), Lübeck, Germany

\author{
Accepted for publication \\ July 13, 2017. \\ Address correspondence to Peter \\ König, M.D., Institut für Anat- \\ omie, Universität zu Lübeck, \\ Ratzeburger Allee 160, 23562 \\ Lübeck, Germany. E-mail: \\ koenig@anat.uni-luebeck.de.
}

\begin{abstract}
Integrity of the airway epithelium is essential for normal lung function. However, studies analyzing the repair process of small epithelial lesions in pseudostratified airway epithelium are missing. To follow airway-epithelial wound closure over time, we lesioned small areas of the mouse tracheal epithelium ( 1 to 12 cells) using a femtosecond laser and followed wound closure up to 6 hours by autofluorescence multiphoton microscopy. Selected lesions were also examined by scanning and transmission electron microscopy and by staining of filamentous actin. Most lesions with a size up to six cells closed by elongation of the surrounding epithelial cells within 6 hours, and all damaged cells were extruded from the epithelium. Electron microscopy confirmed that the surrounding epithelial cells directly closed lesions up to six cells. Most lesions larger than six cells did not close in the observation period of 6 hours, but we observed that basal cells flattened to cover the basement membrane. Delayed wound closure was, in part, attributable to damage of the basement membrane. Cells facing the lesion exhibited increased filamentous actin staining, indicating active cell movement. Not all cells initially facing the lesion participated directly in wound closure, indicating that closure is driven by movement of individual cells rather than a transepithelial coordinated process. Small wounds in the pseudostratified airway epithelium close within hours to preserve epithelial integrity. (Am J Pathol 2017, 187: 2451-2460; http://dx.doi.org/10.1016/j.ajpath.2017.07.006)
\end{abstract}

The airway epithelium acts as a front-line barrier against a large number of environmental factors, like allergens, pathogens, and inhaled particles. Thus, airway epithelial integrity is essential for normal lung function. Inhalation of pathogenic or toxic agents can disturb this integrity by disrupting cell-cell contacts via proteolysis. Also, allergens from house dust mite, cockroach, and fungi have protease activity $^{1}$ and are known to induce epithelial damage. ${ }^{2-6}$ Bacterial pathogens produce lipoproteins that can induce apoptosis, ${ }^{7}$ and viral pathogens, like respiratory syncytial virus, rhinovirus, and influenza virus, have been shown to cause epithelial cell apoptosis. $^{8-11}$ Not all encounters with allergens, bacteria, and viruses result in widespread epithelial destruction, but they lead to localized epithelial damage. ${ }^{12}$ As these local lesions impair epithelial integrity, they have to be repaired quickly to maintain the epithelial barrier and mucociliary clearance function.

The regeneration process of widespread airway epithelial lesions has been well described. Classic models include widespread denudation of the respiratory tract by inhalation of nitrogen dioxide or sulfur dioxide, ${ }^{13-15}$ intraperitoneally applied naphthalene, ${ }^{16-20}$ or manual destruction. ${ }^{21}$ Herein, the regeneration takes place within 2 to 3 weeks by basal cell flattening, proliferation, and differentiation into a ciliated pseudostratified columnar airway epithelium.

However, the regeneration process of small epithelial lesions in the airway epithelium is less clear. Studies in airway cell culture suggest that single apoptotic cells are extruded apically from the epithelial layer by active expulsion by the surrounding epithelial cells. ${ }^{22}$ Tadokoro et $\mathrm{al}^{15}$ showed that, in response to cell crowding, apoptotic cells get extruded from the epithelial layer into the luminal space of the airways. Recent data suggest that apoptotic cells can also be phagocytosed by other airway epithelial cells

Supported by internal funding from the German Center for Lung Research 82DZL00102 (P.K. and G.H.).

Disclosures: None declared. 

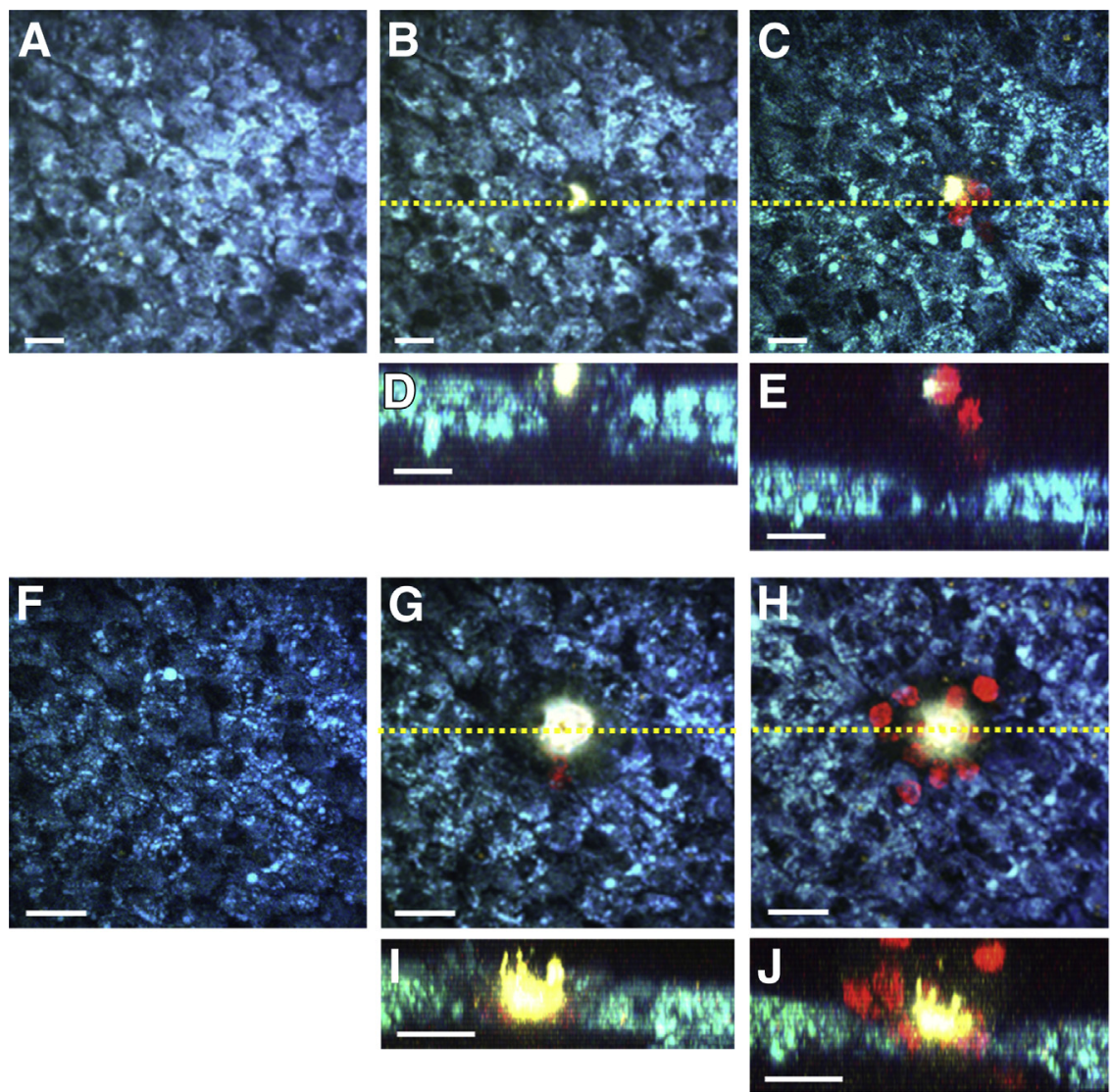

Figure 1 Induction of small lesions by laser irradiation. Multiphoton imaging of the trachea by autofluorescence and propidium iodide staining. En face view of the epithelium $(\mathbf{A}-\mathbf{C}$ and $\mathbf{F}-\mathbf{H})$ and side view of the time points after damaging (D, E, $\mathbf{I}$, and $\mathbf{J})$. Dotted lines in the en face views mark the positions of the side views. Intact epithelium (before lesioning; $\mathbf{A}$ and $\mathbf{F}$ ) is damaged by focusing the laser beam onto a distinct cell for 3 seconds to induce small lesions (B-E) or for 5 to 8 seconds for larger lesions $(\mathbf{G}-\mathbf{J})$. Directly after lesioning, hyperfluorescent cell debris were observed in the area of the laser spot (3 minutes after lesioning; $\mathbf{B}$, D, G, and $\mathbf{I}$ ), and lesioned cells were stained by propidium iodide (C and $\mathbf{G}$ ). Hyperfluorescent cell debris and damaged cells were extruded into the lumen. The epithelium is intact at the side of smaller lesions ( 2 hours 30 minutes after lesioning; $\mathbf{C}$ and $\mathbf{E}$ ), whereas larger lesions still have cell debris and propidium-positive cells in the hole 2 hours after damaging ( $\mathbf{H}$ and $\mathbf{J}$ ). Scale bars $=10$ $\mu \mathrm{m}(\mathbf{A}-\mathbf{J})$. in vivo, ${ }^{23}$ indicating that an alternative pathway for clearance of damaged cells exists.

To date, no studies analyzed the repair processes of small epithelial lesions in the intact tracheal epithelium and followed the fate of the damaged cells and the reaction of the surrounding cells over time.

A well-established tracheal explant model ${ }^{24,25}$ was, therefore, used to induce circumscribed damage of 1 to 12 cells by femtosecond laser irradiation and followed the reaction of damaged and surrounding cells up to 6 hours by autofluorescence multiphoton microscopy. ${ }^{25,26}$

Small lesions up to four cells closed by elongation of the surrounding epithelial cells within 5 hours, and all damaged cells were extruded from the epithelium. Most lesions of five to six cells closed in up to 6 hours. Electron microscopy demonstrated that small lesions up to six cells were closed by the surrounding epithelial cells. In larger lesions, in addition to the response of the surrounding cells, basal cells flattened to cover the basement membrane. Lesions larger than six cells often did not close during the observation period of 6 hours, probably because of additional damage of the basement membrane. Staining with phalloidin demonstrated that the remaining undamaged epithelial cells exhibited accumulation of filamentous actin (F-actin), indicating that the extrusion of the damaged cells is driven by the movement of the surrounding cells. Phagocytosis of damaged epithelial cells by neighboring epithelial cells was not observed.

\section{Materials and Methods}

\section{Animals}

For tracheal imaging, male C57BL/6 6- to 25-week-old mice were used (Charles River Laboratories, Sulzfeld, Germany). All animals were kept according to institutional guidelines, with a 12-hour day-night cycle and food and drink ad libitum. All animal experiments were approved by the Ministry of Energy, Agriculture, the Environment, Nature and Digitalization.

\section{Tissue Preparation}

Tissues were prepared as previously described. ${ }^{25}$ Briefly, mice were euthanized by inhalation of isoflurane (Baxter, Unterschleißheim, Germany). The thorax was opened, and the submandibular gland and the infrahyoid musculature were removed. The trachea was cut caudal to the larynx and cranial to the bifurcation and transferred to a Delta T4 Culture Dish (Bioptechs, Butler, PA). The bottom of the dish was covered with a thin film of Sylgard polymer (Dow Corning, Wiesbaden, Germany) to allow fixation of the tissue with insect needles and was filled with $2 \mathrm{~mL}$ HEPES-buffered Ringer's solution, $\mathrm{pH}$ 7.4. Connective tissue and blood vessels were removed. For imaging the tracheal epithelium with high resolution, the trachea was oriented with the trachealis muscle facing upward. To 


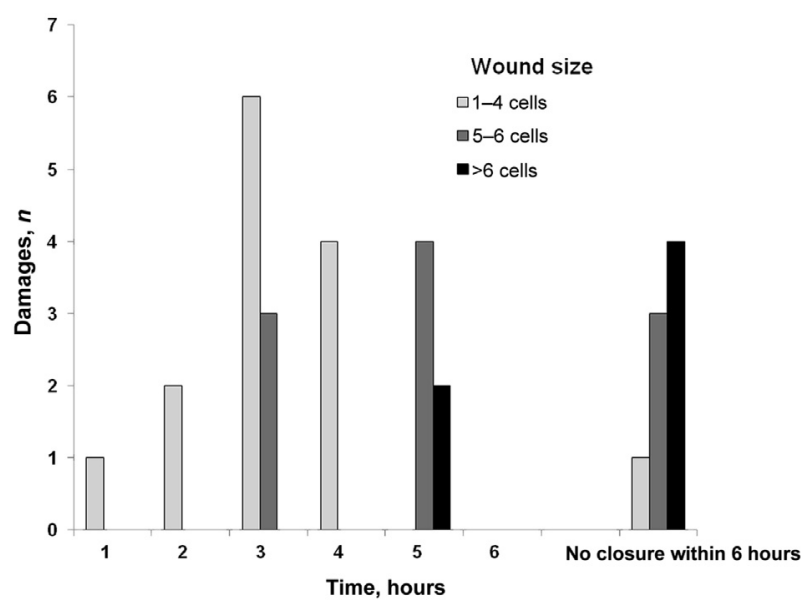

Figure 2 Lesions close over time. Closure times of 30 lesions with different sizes from six experiments were analyzed. Lesions with a size from one to two cells and with a size from three to four cells were grouped together as they behaved similarly.

expose the epithelium, the tracheal muscle was cut. Before imaging, the HEPES-buffered Ringer's solution was replaced with fresh buffer and the culture dish was transferred to the Delta T Stage holder (Bioptechs, Butler, PA), which kept the sample at $37^{\circ} \mathrm{C}$ during the experiment.

\section{Ex Vivo Microscopy}

Imaging was performed with a modified JenLab DermaInspect 101 multiphoton microscope (JenLab GmbH, Jena, Germany) or a TriM Scope II multiphoton microscope (LaVision BioTec GmbH, Bielefeld, Germany).

The JenLab DermaInspect was equipped with a W-PlanApochromat $20 \times$ dip-in objective (numerical aperture $=1.0$;
Carl Zeiss Microscopy GmbH, Jena, Germany) and a tunable femtosecond titanium-sapphire laser (Mai Tai; Newport Spectra-Physics GmbH, Darmstadt, Germany). An excitation wavelength of $750 \mathrm{~nm}$ was chosen for imaging tissue autofluorescence as well as propidium iodide. Emitted light was simultaneously detected in four spectral channels (channel 1, 380 to $450 \mathrm{~nm}$; channel 2,450 to $500 \mathrm{~nm}$; channel 3, 500 to 580 $\mathrm{nm}$; channel 4, 580 to $680 \mathrm{~nm}$ ) by photomultiplier tubes (R1294A and R1295A; Hamamatsu Photonics Deutschland $\mathrm{GmbH}$, Herrsching, Germany). The dichroic filters of the detection module provide a separation between known fluorescence emission spectra of endogenous fluorophores. ${ }^{25} \mathrm{Im}$ ages $(512 \times 512$ pixels) were recorded in either 7.4 or 13.4 seconds. The TriM Scope II multiphoton microscope was equipped with a XLPlan N 25× dip-in objective (numerical aperture $=1.05$; Olympus, Hamburg, Germany) and two titanium-sapphire lasers (Mai Tai HP; Newport SpectraPhysics $\mathrm{GmbH}$ ). Fluorescence was excited at $740 \mathrm{~nm}$, and emitted light was detected in four spectral channels (channel 1, $<435 \mathrm{~nm}$; channel 2, 435 to $495 \mathrm{~nm}$; channel 3, 495 to $560 \mathrm{~nm}$; and channel $4,>560 \mathrm{~nm}$ ) by photomultiplier tubes (H7422-40 and H7422-50; Hamamatsu Photonics Deutschland GmbH).

Time series of a single focal plane, Z-stacks, or Z-stacks over time up to 40 minutes were recorded. In the Z-stacks, the images were taken every $2 \mu \mathrm{m}$. The stacks were processed using IMARIS software version 7.2 (Bitplane, Zurich, Switzerland).

\section{Laser-Induced Lesioning}

With the JenLab DermaInspect, lesions were induced by focusing the titanium-sapphire femtosecond laser beam at
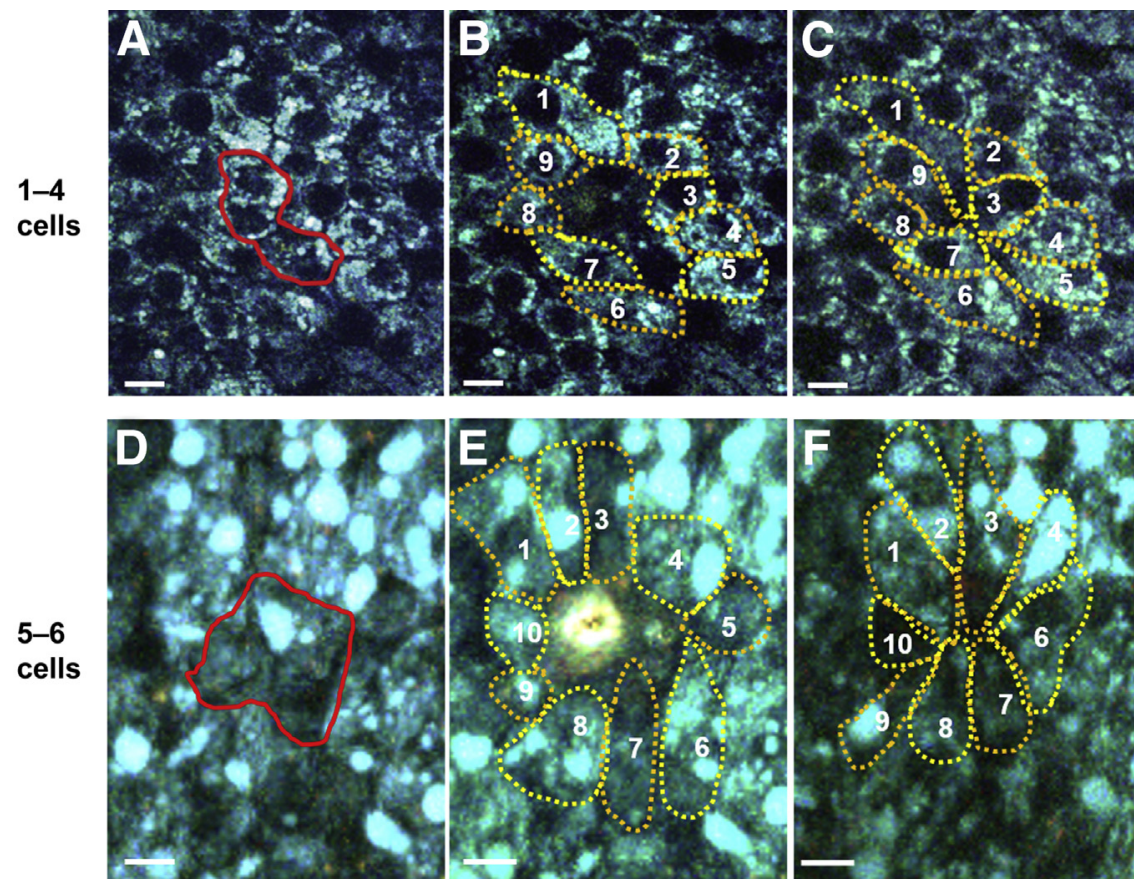

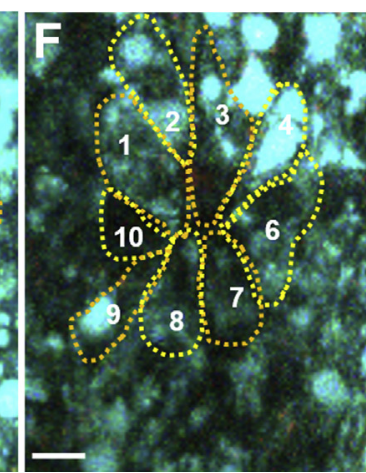

Figure 3 Closure of small lesions, with up to six cells. Multiphoton imaging of the trachea by autofluorescence. En face view of the epithelium. Small lesions of up to six cells close by elongation of the damage-neighboring cells, which stretch and come together in the middle of the lesion. Lesions of one to four cells close faster $(\mathbf{A}-\mathbf{C} ; \mathbf{A}$, before lesioning; B, 3 minutes after lesioning; C, 2 hours after lesioning) than lesions with up to six cells (D-F; D, before lesioning; $\mathbf{E}, 3$ minutes after lesioning; $\mathbf{F}, 4$ hours after lesioning). Red lines in $\mathbf{A}$ and $\mathbf{D}$ mark the cells that are damaged in the experiment. The cells surrounding the damaged area in $\mathbf{B}, \mathbf{C}, \mathbf{E}$, and $\mathbf{F}$ are marked by dotted lines and are numbered (1 to 9 in $\mathbf{B}$ and $\mathbf{C} ; 1$ to 10 in $\mathbf{E}$ and $\mathbf{F}$ ) for better identification. Scale bars $=5 \mu \mathrm{m}(\mathbf{A}-\mathbf{F})$. 

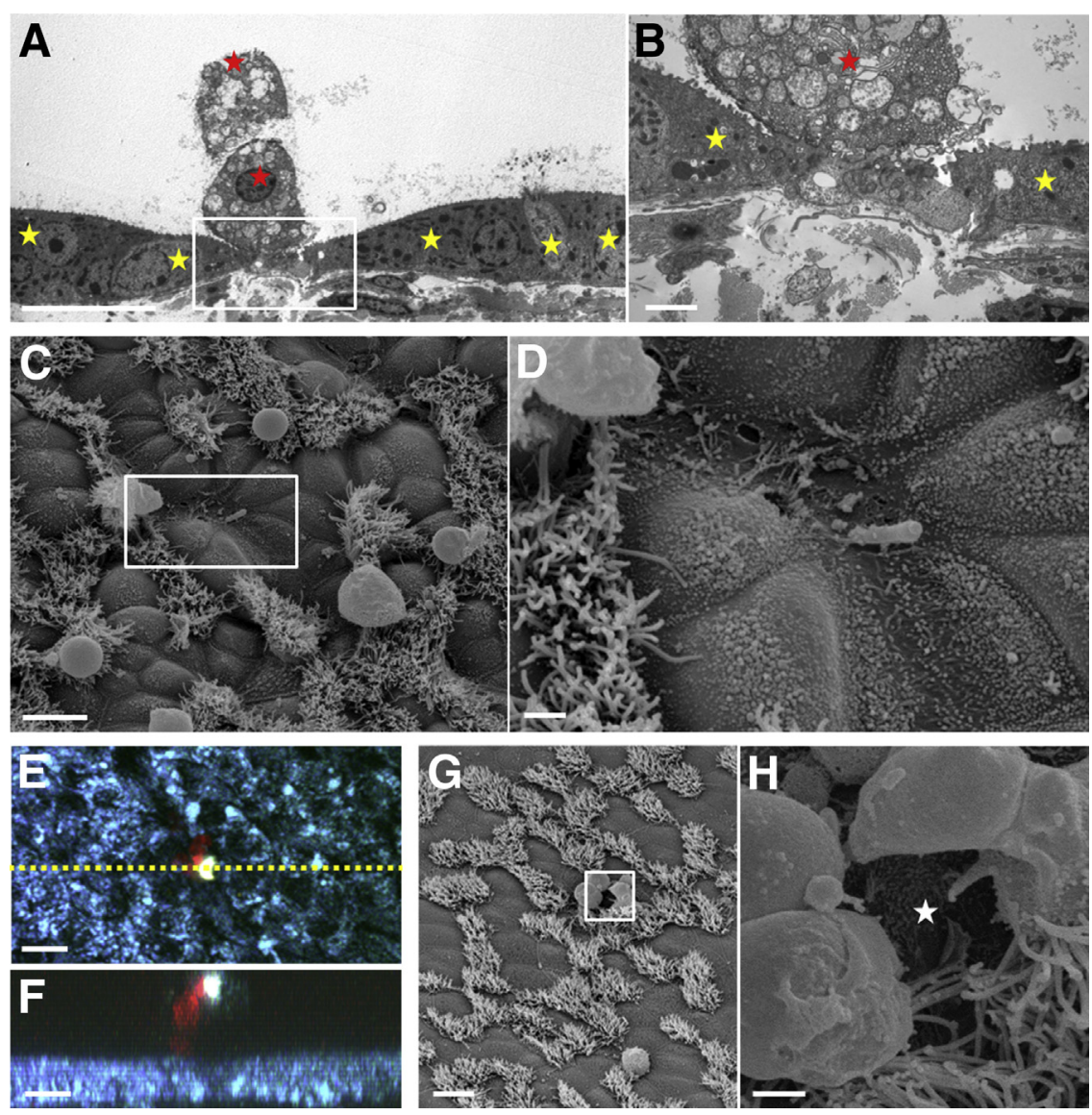

Figure 4 Wound closure of small lesions by surrounding cells. A and B: Transmission electron microscopy of a small lesion 2 hours 30 minutes after lesioning. B: The damaged cells (red stars) were pushed out of the lesion by neighboring columnar epithelial cells and exhibit swollen mitochondria (higher magnification of boxed area in A). Intact epithelial cells (yellow stars) show a stretched morphology. C and D: Scanning electron microscopy images of a closed lesion 7 hours after lesioning. Tips of elongated cells meet at the lesion site. D: Higher magnification of the boxed area in C. E-H: Small lesion after closure. The lesioned epithelium was observed by multiphoton microscopy (E and $\mathbf{F}) 2$ hours after lesioning [en face view (E) and side view (F)] and was fixed 4 hours after lesioning for scanning electron microscopy ( $\mathbf{G}$ and $\mathbf{H})$. The hyperfluorescent cell debris and propidium iodide-positive cells (red) are extruded ( $\mathbf{E}$ and $\mathbf{F}$ ). F: The dotted line marks the location of the side view. The extruded cells lay on top of the epithelium 4 hours after damaging ( $\mathbf{G}$ and $\mathbf{H})$. $\mathbf{H}$ : Higher magnification of the boxed area in $\mathbf{G}$ indicates that the epithelium is intact under the cell debris. The intact cell is marked by a white star. Scale bars: $10 \mu \mathrm{m}(\mathbf{A}, \mathbf{C}$, and $\mathbf{E}-\mathbf{G}) ; 2 \mu \mathrm{m}$ (B, $\mathbf{D}$, and $\mathbf{H})$.
$81 \mathrm{~mW}$ radiant flux at the exit of the objective to single epithelial cells for 2 to 8 seconds. With the TriM Scope multiphoton microscope, a distinct small scan field with one to six cells (10 to $40 \mu^{2}$ ) was scanned, with a high laser power of 50 to $55 \mathrm{~mW}$ exiting the objective for 2 to 5 seconds to damage the cells in this focused area. No differences in lesions were detected by optical imaging and electron microscopy between the two microscopes.

Propidium iodide (BD Biosciences, Heidelberg, Germany; $0.1 \mu \mathrm{g} / \mathrm{mL}$ ) was present in the bath solution throughout the experiment to stain damaged cells.

\section{EdU Proliferation Assay}

5-Ethynyl-2'-deoxyuridine (EdU) is a nucleoside analog of thymidine whose incorporation can be used to label cells undergoing DNA replication. Proliferating cells in the trachea were evaluated by using the Click-iT EdU Alexa Fluor 594 Imaging Kit (Invitrogen, Dreieich, Germany), according to the manufacturer's instructions. Briefly, after laser-induced lesioning of tracheal epithelial cells, the tissue was incubated with $10 \mu \mathrm{mol} / \mathrm{L} \mathrm{EdU}$ for 2,4 , or 6 hours at $37^{\circ} \mathrm{C}$, then fixed with $3.7 \%$ formaldehyde for 15 minutes, and treated with $0.5 \%$ Triton X-100 for 20 minutes at room temperature. After washing twice with phosphate-buffered saline, the cells were reacted with Click-iT reaction cocktail for 30 minutes.
Subsequently, cell nuclei were stained with Hoechst 33342 (Invitrogen), and tracheae were imaged by multiphoton microscopy.

\section{Preparing Tissue and Sections for Transmission Electron and Scanning Electron Microscopy}

For light and transmission electron microscopy, tracheae were fixed after selected time points in Monti's fixative (2\% glutardialdehyde, $0.6 \%$ paraformaldehyde, and $0.03 \% \mathrm{CaCl}_{2}$ in $0.06 \mathrm{~mol} / \mathrm{L}$ cacodylate buffer, $\mathrm{pH} \mathrm{7.35)}$ at least overnight and transferred to $0.1 \mathrm{~mol} / \mathrm{L}$ cacodylate buffer, followed by incubation with $1 \%$ osmium tetroxide in cacodylate buffer overnight. After washing in cacodylate buffer, tissue was dehydrated by incubating it in increasing concentrations of ethanol, transferred to propylene oxide, and left in a 1:1 mixture of Araldite M (Sigma-Aldrich, Munich, Germany) and propylene oxide overnight. Then, it was transferred to Araldite M for 30 minutes, followed by fresh Araldite M. For polymerization, tissue was incubated for at least 2 days at $58^{\circ} \mathrm{C}$. Semithin sections (700 to $800 \mathrm{~nm}$ thick) were cut using an ultramicrotome (Ultracut E; Leica Microsystems, Wetzlar, Germany), stained with Richardson's stain for plastic sections, ${ }^{27}$ coverslipped with DePeX, and analyzed using conventional light microscopy (Axioskop 2 plus; Carl Zeiss Microscopy GmbH, Jena, Germany). 

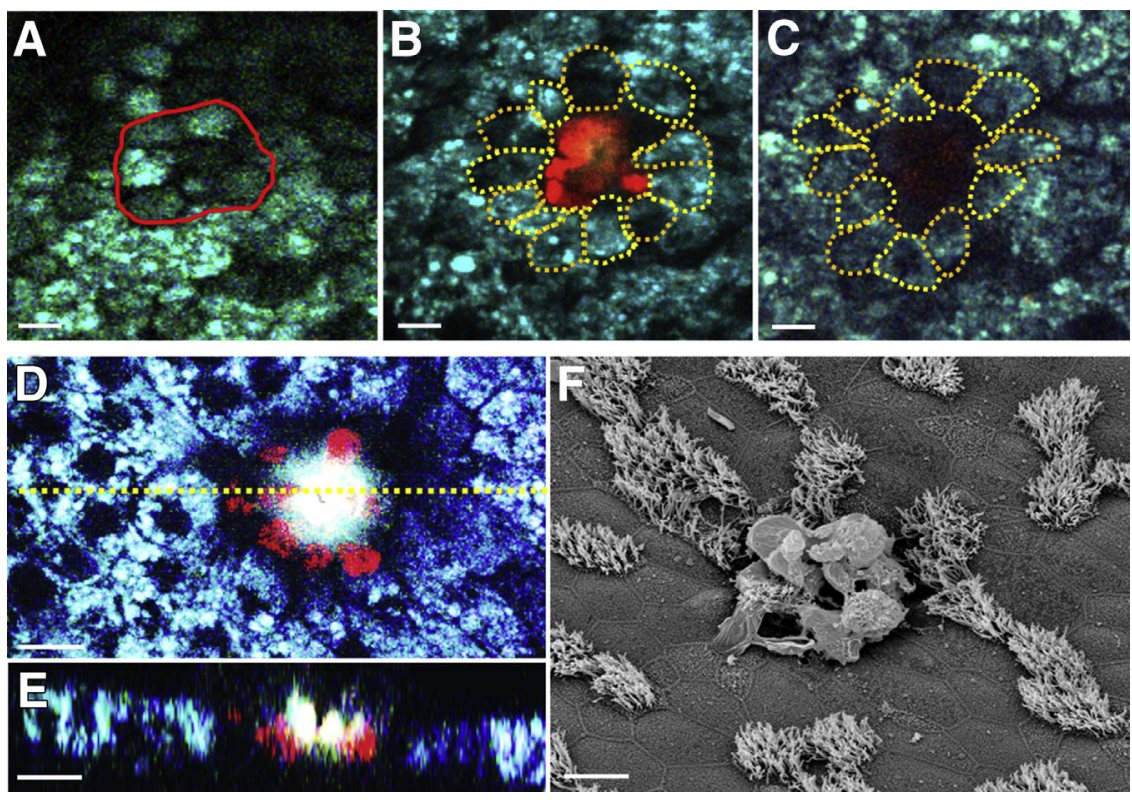

Figure 5 Larger lesions often do not close within the observation time. A-C: Multiphoton microscopy of a lesion with more than six cells. Cells indicated with a red circle (A) (before lesioning) are damaged. Dotted lines [15 minutes (B) and 6 hours (C) after lesioning] frame the cells immediately adjacent to the damaged cells. D-F: Large lesion with more than six cells. After multiphoton imaging of the trachea, scanning electron microscopy was performed. En face view of the basal part (D) and side view of the lesion (E) 2 hours after lesioning. The dotted line in $\mathbf{D}$ marks the location of the side view (E). Damaged cells stained by propidium iodide appear red. Scanning electron micrograph of the same lesion shown in $\mathbf{D}$ and $\mathbf{E} 2$ hours 30 minutes after lesioning (F). Scale bars $=10 \mu \mathrm{m}(\mathbf{A}-\mathbf{F})$.
Ultrathin sections were cut at approximately $80 \mathrm{~nm}$ and were transferred to copper grids. Sections were contrasted in a contrasting system for ultrathin sections (Leica EM AC20; Leica Microsystems) using uranyl acetate ready-to-use solution (Leica Microsystems), followed by lead citrate ready-to-use-solution (Leica Microsystems), and were subsequently evaluated using a transmission electron microscope (Jeol JEM 1011; JEOL, Eching, Germany).

For scanning electron microscopy, tracheae were fixed in Monti's fixative at least overnight at $4^{\circ} \mathrm{C}$ and then transferred to cacodylate buffer, followed by incubation with $1 \%$ osmium tetroxide in cacodylate buffer overnight. After washing in cacodylate buffer, tissue was dehydrated by incubating in increasing concentrations of ethanol.
Afterward, the tissue was critical point dried. The dried samples were glued on a sample holder and sputtered with gold. Samples were imaged using a scanning electron microscope (Philips SEM 505; Philips, Eindhoven, the Netherlands).

\section{Whole-Mount Staining of Filamentous Actin}

After induction of epithelial lesions and multiphoton microscopy, tracheae were transferred to a cork tile (approximately $15 \times 10 \times 2 \mathrm{~mm}$ ) and were positioned using insect needles. Afterward, the tile was transferred upside down into $4 \%$ phosphate-buffered saline-buffered paraformaldehyde $(\mathrm{pH}$ 7.4) for 1 to 12 hours for fixation. Then, the tracheae were
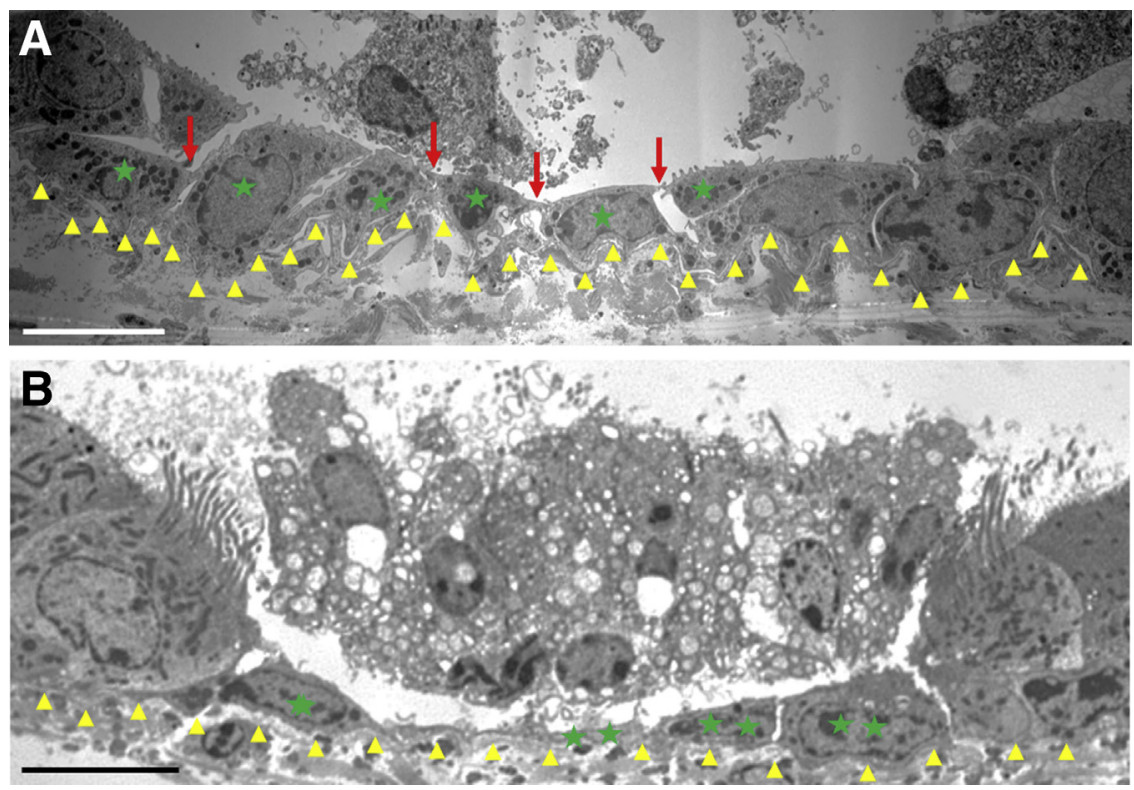

Figure 6 Deformation of basal cells in lesions with more than six cells. Transmission electron microscopy of lesions with more than six cells. A: Basal cells (green stars) cover the wound and are interconnected (red arrows) 3 hours after lesioning. B: Basal cells (green stars) flattened to cover the basement membrane 2 hours 30 minutes after lesioning. Yellow arrowheads point to the basement membrane. Scale bars $=10 \mu \mathrm{m}(\mathbf{A}$ and $\mathbf{B})$. 


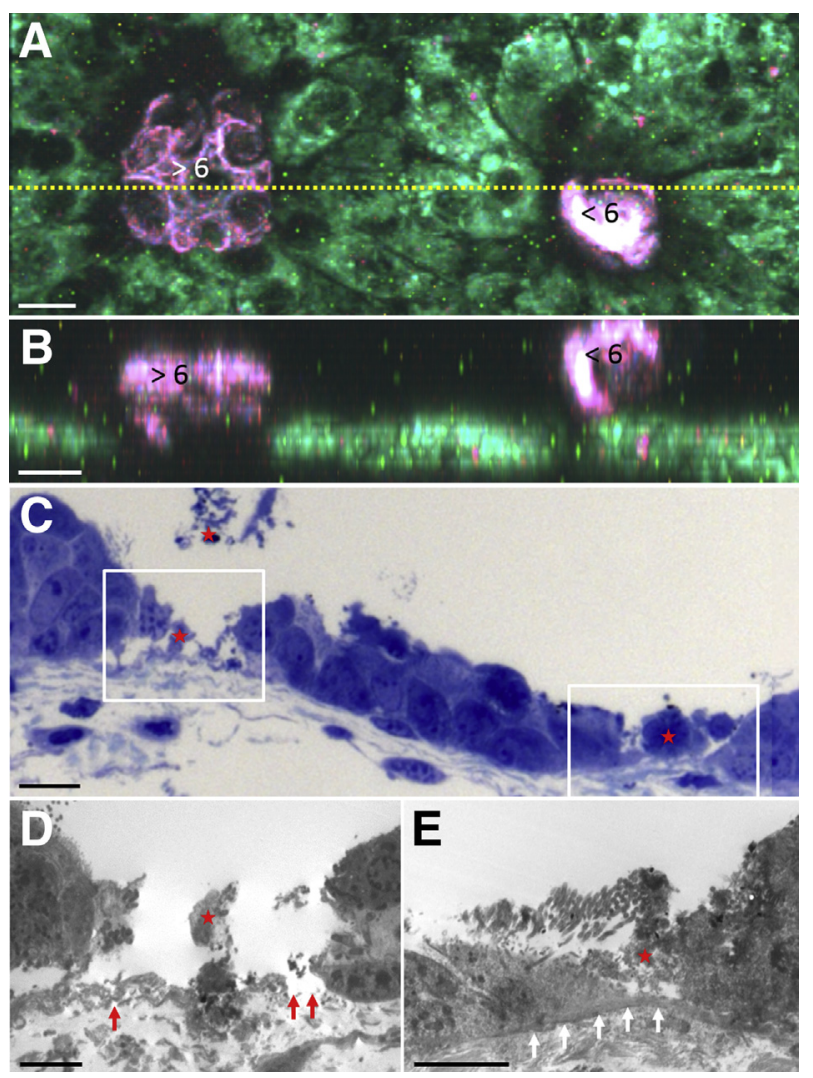

Figure 7 Larger lesions often show a damaged basement membrane. A: En face of the airway epithelium with intact cells (green), a lesion with more than six cells, and a lesion with fewer than six cells 2 hours 30 minutes after lesioning with hyperfluorescent cell debris (white and pink) in the lesioned area. B: Side view of the two lesions 2 hours 30 minutes after lesioning. The dotted line in A marks the location of the side view. C: Semithin section of the same area 3 hours after lesioning. Cell debris are visible over or in the lesion (red stars). D: Electron microscopic image of the left boxed area in C. E: Electron microscopic image of the right boxed area in $\mathbf{C}$. The basement membrane (red arrows) in the larger lesion (D) is not intact, whereas in the smaller lesion (E) the basement membrane (white arrows) is intact and the lesion is nearly closed 3 hours after lesioning. Red stars mark the cell debris. Scale bars: $10 \mu \mathrm{m}(\mathbf{A}-\mathbf{C}) ; 5 \mu \mathrm{m}$ (D and $\mathbf{E})$.

rinsed three times for 15 minutes with phosphate-buffered saline and incubated with phalloidin conjugated to Atto 550 (1:10,000; Sigma-Aldrich). After rinsing with phosphatebuffered saline for three times for 15 minutes, tracheae were mounted between coverslips using buffered Mowiol-4-88 (Sigma Aldrich). Whole mounts were imaged using a confocal laser scanning microscope (510; Carl Zeiss Microscopy $\mathrm{GmbH}$ ). Damaged areas were identified by their strong autofluorescence and/or by overlaying the multiphoton images with the confocal images.

\section{Results}

Femtosecond-Laser Irradiation Can Be Used to Lesion the Airway Epithelium

The laser beam of the titanium-sapphire laser can be used to induce small lesions (Figure 1, A-E), comprising one to four cells, or by increasing scan duration, up to 12 cells (Figure 1, F-J). Lesions were visible by hyperfluorescent cell debris (Figure 1, B-E and G-J). Depending on the size of the lesion, a loss of the autofluorescent signal in the cells surrounding the hyperfluorescent debris was sometimes observed (Figure 1, G-J). Propidium iodide in the bath solution stained the nuclei of damaged cells (Figure 1, C, E, and $\mathrm{G}-\mathrm{J})$. The cell(s) in the laser focus were immediately propidium iodide positive, and damaged surrounding cells were stained during the following hour. Larger lesions contained up to four to six propidium iodide-positive cells, and the number increased during the observation time.

\section{Time Course of Lesion Closure}

Of all lesions up to four cells, $93 \%$ closed within 4 hours. After 6 hours, $70 \%$ of lesions with five to six damaged cells closed. In contrast, $67 \%$ of lesions larger than six cells did not close during the observation period (Figure 2).

\section{Lesions with the Size of Up to Six Cells Are Closed by Neighboring Cells}

Observation of the time course of lesion closure revealed that in lesions up to six cells, the closure was mainly driven by the surrounding cells. The surrounding cells changed their shape and elongated to close the lesion (Figure 3). During the protrusion of the surrounding cells, the damaged cells were extruded from the epithelium and were quickly removed from the extrusion site, most likely by cilia-driven fluid flow (Supplemental Video S1). Electron microscopy confirmed that the surrounding columnar epithelial cells closed the wound by cell protrusions (Figure 4, A-D). In some lesions, epithelial cells were not removed and remained close to the lesion site. However, scanning electron microscopy confirmed data from multiphoton microscopy that lesions were closed even if the cell debris was not removed (Figure 4, E-H).

\section{In Lesions Larger than Six Cells, Basal Cells Participate in Wound Closure}

In lesions that did not close during the observation period of 6 hours, the surrounding cells largely remained in their position within the epithelium and exhibited less change of their shape (Figure 5, A-E). The cell debris often remained close to the lesion site, and the surface epithelium was not able to close the lesion within the observation period. Analysis of damaged areas by scanning electron microscopy confirmed only limited participation of surrounding epithelial cells (Figure 5F). Analysis of larger lesions with transmission electron microscopy indicated that, in many experiments, only the surface epithelium was affected, and the basal epithelial cells covered the basement membrane at the lesion site (Figure 6). In some experiments, it was confirmed that, in addition to surface epithelial cells, the 

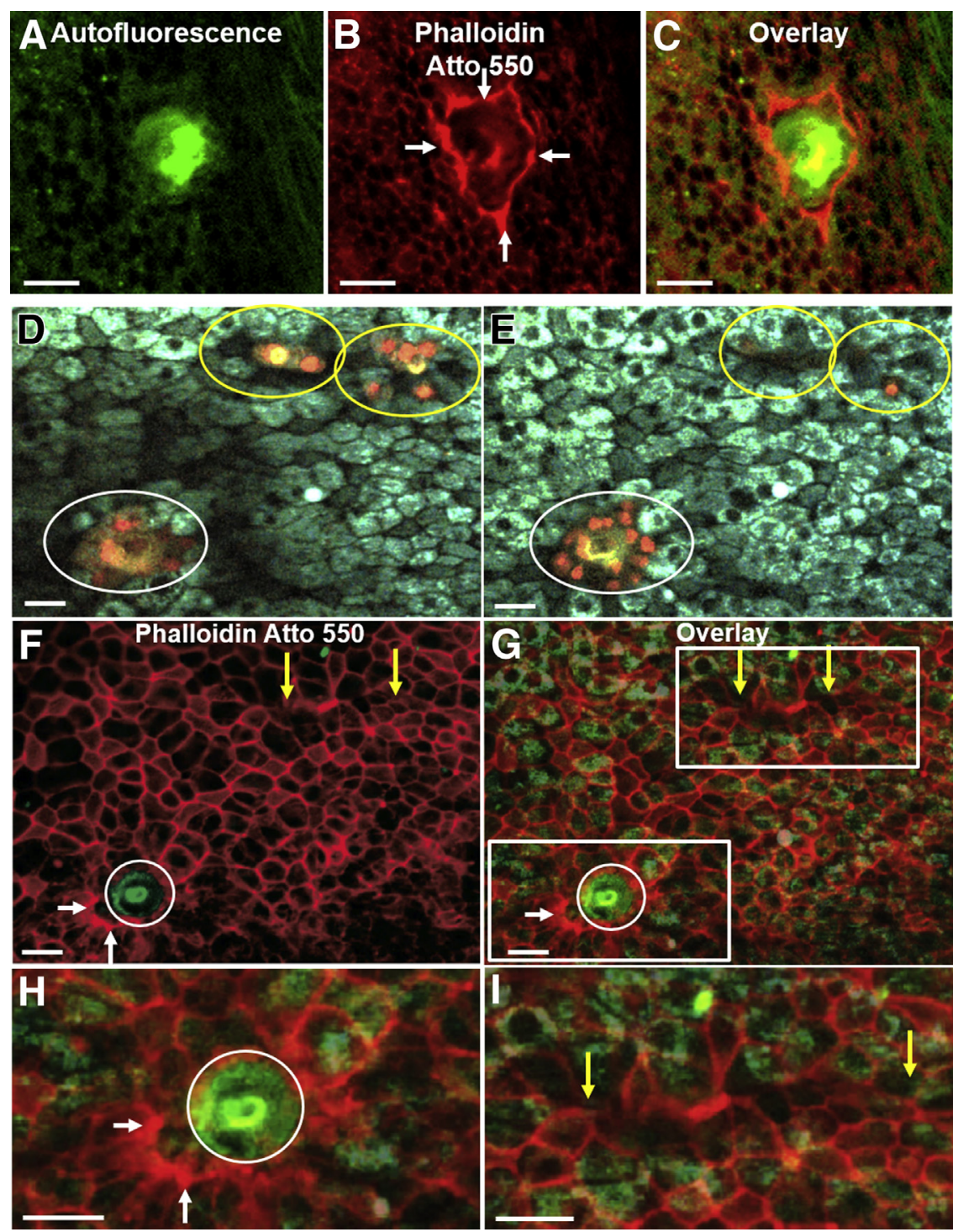

Figure 8 Epithelial cells contacting damaged cells show filamentous actin staining. A-C: Staining the trachea with phalloidin Atto 550 (red) 2 hours after multiphoton microscopy. Hyperfluorescent cell debris are visible by their autofluorescence (green). Columnar epithelial cells contacting damaged cells are phalloidin positive in areas facing damaged cells (B) (white arrows). D and $\mathbf{E}$ : Lesions (large lesion indicated by white circles; small lesions, yellow circles) made by multiphoton microscopy 20 minutes (D) and 1 hour 45 minutes (E) after lesioning. Damaged cells were stained by propidium iodide (red). F: Phalloidin Atto 550 staining of the trachea after multiphoton microscopy at 2 hours 15 minutes after lesioning. G-I: Overlay of the confocal laser scanning microscope image and the multiphoton image at the end of the experiment (green) with phalloidin Atto 550 staining (red) 2 hours 15 minutes after lesioning. Bottom boxed area in $\mathbf{G}$ is shown at higher magnification in $\mathbf{H}$; top boxed area, in I. Cells surrounding the large lesion (white circles in D-H) exhibit phalloidin staining (white arrows in $\mathbf{F}-\mathbf{H}$ ), whereas no phalloidin staining surrounding the former small lesions (yellow circles in $\mathbf{D}$ and $\mathbf{E}$ and yellow arrows in $\mathbf{F}$, $\mathbf{G}$, and $\mathbf{I})$ is detectable. Scale bar $=20 \mu \mathrm{m}(\mathbf{A}-\mathbf{I})$. laser damage affected the basal cells and the basement membrane (Figure 7).

\section{Epithelial Cells Adjacent to Damaged Cells Exhibit F-Actin Staining}

Phalloidin staining indicated that F-actin accumulates in the part of the surrounding epithelial cells that is immediately adjacent to the damaged cells (Figure 8, A-C) or that participates in lesion closure (Supplemental Video S2). In areas where lesion closure is in progress, increased F-actin can be detected, whereas in areas where lesion closure is completed, no increased F-actin staining was detectable (Figure 8, D-I).

\section{Effect of Proliferating Cells on Wound Closure}

To investigate the effect of proliferation on wound closure after laser-induced lesioning, the tissue was incubated with EdU for 2, 4, and 5 hours after laser treatment. At each time point, 6 to 12 lesions of different sizes were examined. The cells directly surrounding the lesioned region showed no proliferation (Figure 9). In other areas of the trachea, some, albeit few, proliferating cells could consistently be observed.

\section{Discussion}

Using the femtosecond laser of the multiphoton microscope, we were able to induce small lesions in the airway epithelium. Autofluorescence-based multiphoton microscopy allowed us to observe the immediate healing process of the generated wounds. After lesioning, the surrounding columnar epithelial cells actively protruded underneath the damaged cells, leading to their extrusion. In lesions that could not be closed directly by the surrounding cells, basal cells reacted to cover the basement membrane. Only the minority of lesions larger than six cells were closed by 

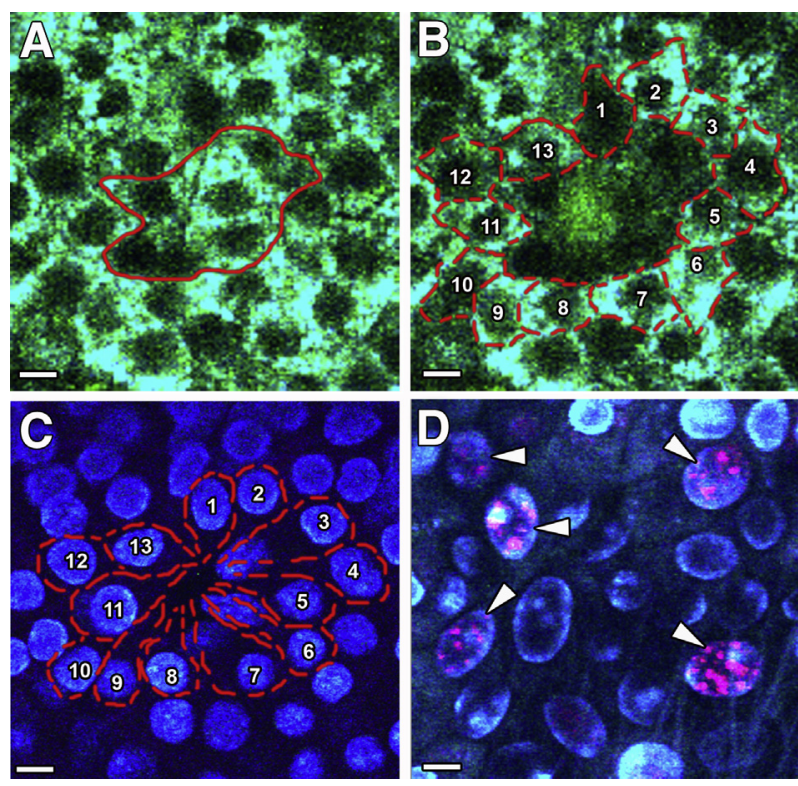

Figure 9 Effect of proliferation on wound closure. A and B: Multiphoton imaging of tracheal epithelium before $(\mathbf{A})$ and 3 minutes after (B) damaging. The red lines in $\mathbf{A}$ mark the cells that were damaged and lost autofluorescence in B. B and C: The cells surrounding the damaged area are marked by dashed lines and are numbered (1 to 13$)$ for better identification. C: The same area as in B after incubation with 5-ethynyl-2'-deoxyuridine (EdU) for 5 hours and subsequent fixation and labeling of EdU and Hoechst 33342 (blue). Because of the loss of mitochondrial NAD(P)H autofluorescence after fixation, the cell borders are no longer clearly visible, but only cell nuclei were stained (blue). The Click-iT reaction reveals no EdU staining of cell nuclei in the area around the lesion. D: Positive EdU staining (purple) is observed in proliferating cell nuclei at different areas unrelated to laser damage (arrowheads). Scale bars: $10 \mu \mathrm{m}$ (A-C); $5 \mu \mathrm{m}$ (D).

surface epithelium during the observation period of 6 hours. This was most likely because of the fact that the lesions were too large for direct closure and that the basement membrane was damaged in some experiments. Our data clearly indicate that small lesions in the airway pseudostratified epithelium effectively heal within hours, giving pathogens only a limited access to the basement membrane.

In our experiments, in small lesions, the extrusion of the damaged epithelial cells was driven by the surrounding epithelial cells. These cells elongated, and their protrusions moved underneath the damaged cells. The damaged cells were either removed or remained on the epithelium. It is most likely that fluid flow, generated by ciliary beating, removed the damaged cells. The existence of fluid flow in the trachea that is able to remove particles and cells was shown by our group earlier in a similar ex vivo model. ${ }^{24}$ Although it was proposed earlier that epithelial cells are able to phagocytose other epithelial cells, ${ }^{23}$ phagocytosis of damaged cells by neighboring epithelial cells was never observed. Whether professional phagocytes ultimately clear the remaining cells on the epithelium or phagocytosis by epithelial cells occurs at a later time point remains to be determined.
The observed reaction of neighboring cells to extrude damaged cells from the epithelial layer has been observed repeatedly in cell culture, ${ }^{15,22}$ and was also described in the intestine and the stomach as well as during embryogenesis. $^{28,29}$ Although cell cultures and gut epithelium are single-layered epithelia, which lack basal cells, the mechanism in pseudostratified airway epithelium seems to work similarly. In single-layered epithelia, cells around a damaged cell express an actin ring that surrounds the damaged cells. F-actin staining was also observed in the tracheal columnar epithelial cells that were immediately adjacent to the lesioned cells.

In cell culture experiments, the term purse-string was coined for this phenomenon because the phalloidin staining has the appearance of a transcellular actin ring. The phenomenon of a purse-string of actin or an actin-myosin ring, which closed lesions by contraction and thereby stretching the cells of the border together, can be found conserved in vertebrates, fish, and insects. ${ }^{30}$ However, this term implies that this actin purse-string actively contracts and drags the remaining parts of the cells toward closing the lesion. In migrating cells, phalloidin also stains the leading edge of cells. Watson et $\mathrm{al}^{31}$ and Bullen et $\mathrm{al}^{32}$ showed that lamellipodia and filopodia were observed together with the building of an actin purse-string in mouse and human tissue. Thus, an alternative explanation for the occurrence of the purse-string staining pattern is that all cells adjacent to the lesion move independently. This hypothesis is supported by the fact that perfect purse-strings were not observed in larger lesions, but sometimes the phalloidin staining was either not continuous or found between two parts of the cells that were not in direct contact to the damaged cells. Furthermore, pulling on a purse-string would generate a circular pattern on closure, but a more longitudinal staining where wounds had recently been closed or were about to close was often observed. A study of Bement et $\mathrm{al}^{33}$ showed, in Caco-2 cell culture, that in lesions larger than eight cells, the actin staining had the form of arcs and not of a ring. In these lesions, more lamellipodia extended to close the wound, confirming our observations.

In contrast to single-layer epithelia, the pseudostratified epithelium harbors basal cells that have been shown to be instrumental in closing larger wounds because of sulfur dioxide - or naphthalene-induced epithelial denudation of columnar epithelial cells in the mouse trachea. ${ }^{14,15,20}$ In these models, the cuboidal basal cells flattened to cover the basement membrane and reseal the epithelium. The same behavior of basal cells was observed after larger epithelial lesions, where the damage area was too big to be immediately closed by surrounding columnar epithelial cells. Erjefält et $\mathrm{al}^{21,34}$ described a similar phenomenon of basal cell flattening in vivo after a selective loss of columnar cells in their experiments with tracheae of guinea pigs. Basal cells normally have a drop-shaped morphology and present $5 \%$ to $10 \%$ of the mouse tracheal epithelium. ${ }^{35}$ But in our experiments, they showed a cuboidal shape at the borders of the 
lesions and built a closed layer of flattened cells onto the basement membrane at the floor of the damage after 2.5 hours. It is known that basal cells proliferate and differentiate to rebuild an airway epithelium after large lesions. ${ }^{20,34}$ Proliferation of either basal or epithelial cells was not detected in the observation time, but our data confirm that basal cells also participate in the immediate closure of smaller lesions.

Whether, in our experiments, the secondary closure of these lesions is because of migration of columnar epithelial cells or proliferation and differentiation of basal cells remains to be determined.

Small lesions, comprising up to four cells, are closed, on average, after 2 hours, and lesions with five to six cells are closed after 4 hours. Larger lesions most often did not close well at 6 hours. Electron microscopy revealed that lesions that did not close well often contained lesions of the basement membrane. An intact basement membrane facilitates adhesion and migration of cells and regulates the phenotype and polarity as well as apoptosis. ${ }^{36,37}$ Thus, the fact that the basement membrane was affected in larger lesions might explain their incomplete closure.

Our current data are restricted to mouse tracheal tissue that is regarded as a model for small human intrapulmonary airways. However, further studies are needed to confirm that the same processes are present in human airways. There is no general hindrance to study intrapulmonary airways by multiphoton microscopy, provided that the tissue can be obtained shortly after removal. Using the same setup, we previously successfully imaged explanted nasal airways. ${ }^{25}$

In conclusion, our data show that the airway epithelium can close small lesions in the epithelium within a few hours by extension of the surrounding epithelial cells and extrusion of the damaged cells into the lumen. The time course of the closure of the lesion is comparable to closure times observed in the gut in vivo. ${ }^{38}$ This indicates an effective mechanism by which the epithelial cells are able to maintain epithelial integrity or by which the break in epithelial integrity can be kept to a minimum. The mechanism observed follows a well-described pattern already observed in cell culture and in the gut. In case of lesions that cannot be closed directly by columnar epithelial cells, basal cells can seal the basement membrane to maintain barrier integrity.

\section{Acknowledgment}

We thank Gudrun Knebel for expert technical assistance.

\section{Supplemental Data}

Supplemental material for this article can be found at http://dx.doi.org/10.1016/j.ajpath.2017.07.006.

\section{References}

1. Jacquet A: Interactions of airway epithelium with protease allergens in the allergic response. Clin Exp Allergy 2011, 41:305-311

2. Asokananthan N, Graham PT, Stewart DJ, Bakker AJ, Eidne KA, Thompson PJ, Stewart GA: House dust mite allergens induce proinflammatory cytokines from respiratory epithelial cells: the cysteine protease allergen, Der p 1, activates protease-activated receptor (PAR)2 and inactivates PAR-1. J Immunol 2002, 169:4572-4578

3. Nathan AT, Peterson EA, Chakir J, Wills-Karp M: Innate immune responses of airway epithelium to house dust mite are mediated through beta-glucan-dependent pathways. J Allergy Clin Immunol 2009, 123:612-618

4. Heijink IH, van Oosterhout A, Kapus A: Epidermal growth factor receptor signalling contributes to house dust mite-induced epithelial barrier dysfunction. Eur Respir J 2010, 36:1016-1026

5. Mattila P, Joenväärä S, Renkonen J, Toppila-Salmi S, Renkonen R: Allergy as an epithelial barrier disease. Clin Transl Allergy 2011, 1:5

6. Post S, Nawijn MC, Hackett TL, Baranowska M, Gras R, van Oosterhout AJ, Heijink IH: The composition of house dust mite is critical for mucosal barrier dysfunction and allergic sensitisation. Thorax 2012, 67:488-495

7. Aliprantis AO, Yang RB, Mark MR, Suggett S, Devaux B, Radolf JD, Klimpel GR, Godowski P, Zychlinsky A: Cell activation and apoptosis by bacterial lipoproteins through toll-like receptor-2. Science 1999, 285:736-739

8. Kotelkin A, Prikhod'ko EA, Cohen JI, Collins PL, Bukreyev A: Respiratory syncytial virus infection sensitizes cells to apoptosis mediated by tumor necrosis factor-related apoptosis-inducing ligand. J Virol 2003, 77:9156-9172

9. Othumpangat S, Gibson LF, Samsell L, Piedimonte G: NGF is an essential survival factor for bronchial epithelial cells during respiratory syncytial virus infection. PLoS One 2009, 4:e6444

10. Deszcz L, Gaudernak E, Kuechler E, Seipelt J: Apoptotic events induced by human rhinovirus infection. J Gen Virol 2005, 86: $1379-1389$

11. Maruoka S, Hashimoto S, Gon Y, Nishitoh H, Takeshita I, Asai Y, Mizumura K, Shimizu K, Ichijo H, Horie T: ASK1 regulates influenza virus infection-induced apoptotic cell death. Biochem Biophys Res Commun 2003, 307:870-876

12. Erjefält JS, Korsgren M, Nilsson MC, Sundler F, Persson CG: Prompt epithelial damage and restitution processes in allergen challenged guinea-pig trachea in vivo. Clin Exp Allergy 1997, 27:1458-1470

13. Kawakami M, Yasui S, Yamawaki I, Katayama M, Nagai A, Takizawa T: Structural changes in airways of rats exposed to nitrogen dioxide intermittently for seven days: comparison between major bronchi and terminal bronchioles. Am Rev Respir Dis 1989, 140: $1754-1762$

14. Tadokoro T, Wang Y, Barak LS, Bai Y, Randell SH, Hogan BL: IL6/STAT3 promotes regeneration of airway ciliated cells from basal stem cells. Proc Natl Acad Sci U S A 2014, 111:E3641-E3649

15. Tadokoro T, Gao X, Hong CC, Hotten D, Hogan BL: BMP signaling and cellular dynamics during regeneration of airway epithelium from basal progenitors. Development 2016, 143:764-773

16. Stripp BR, Maxson K, Mera R, Singh G: Plasticity of airway cell proliferation and gene expression after acute naphthalene injury. Am J Physiol 1995, 269:L791-L799

17. Van Winkle LS, Gunderson AD, Shimizu JA, Baker GL, Brown CD Gender differences in naphthalene metabolism and naphthaleneinduced acute lung injury. Am J Physiol Lung Cell Mol Physiol 2002, 282:L1122-L1134

18. Yildirim AO, Veith M, Rausch T, Müller B, Kilb P, Van Winkle LS, Fehrenbach H: Keratinocyte growth factor protects against Clara cell injury induced by naphthalene. Eur Respir J 2008, 32:694-704

19. Oliver JR, Kushwah R, Wu J, Cutz E, Yeger H, Waddell TK, Hu J: Gender differences in pulmonary regenerative response to naphthalene- 
induced bronchiolar epithelial cell injury. Cell Prolif 2009, 42: 672-687

20. Cole BB, Smith RW, Jenkins KM, Graham BB, Reynolds PR, Reynolds SD: Tracheal basal cells: a facultative progenitor cell pool. Am J Pathol 2010, 177:362-376

21. Erjefält JS, Erjefält I, Sundler F, Persson CG: In vivo restitution of airway epithelium. Cell Tissue Res 1995, 281:305-316

22. Gu Y, Forostyan T, Sabbadini R, Rosenblatt J: Epithelial cell extrusion requires the sphingosine-1-phosphate receptor 2 pathway. J Cell Biol 2011, 193:667-676

23. Juncadella IJ, Kadl A, Sharma AK, Shim YM, Hochreiter-Hufford A, Borish L, Ravichandran KS: Apoptotic cell clearance by bronchial epithelial cells critically influences airway inflammation. Nature 2013, 493:547-551

24. Bermbach S, Weinhold K, Roeder T, Petersen F, Kugler C, Goldmann T, Rupp J, König P: Mechanisms of cilia-driven transport in the airways in the absence of mucus. Am J Respir Cell Mol Biol 2014, 51:56-67

25. Kretschmer S, Pieper M, Hüttmann G, Bölke T, Wollenberg B, Marsh LM, Garn H, König P: Autofluorescence multiphoton microscopy for visualization of tissue morphology and cellular dynamics in murine and human airways. Lab Invest 2016, 96:918-931

26. Orzekowsky-Schroeder R, Klinger A, Martensen B, Blessenohl M, Gebert A, Vogel A, Hüttmann G: In vivo spectral imaging of different cell types in the small intestine by two-photon excited autofluorescence. J Biomed Opt 2011, 16:116025

27. Richardson KC, Jarett L, Finke EH: Embedding in epoxy resins for ultrathin sectioning in electron microscopy. Stain Technol 1960, 35: $313-323$

28. Marchiando AM, Shen L, Graham WV, Edelblum KL, Duckworth CA, Guan Y, Montrose MH, Turner JR, Watson AJ: The epithelial barrier is maintained by in vivo tight junction expansion during pathologic intestinal epithelial shedding. Gastroenterology 2011, 140:1208-1218. $\mathrm{e} 1-\mathrm{e} 2$
29. Aihara E, Hentz CL, Korman AM, Perry NP, Prasad V, Shull GE, Montrose MH: In vivo epithelial wound repair requires mobilization of endogenous intracellular and extracellular calcium. J Biol Chem 2013, 288:33585-33597

30. Garcia-Fernandez B, Campos I, Geiger J, Santos AC, Jacinto A: Epithelial resealing. Int J Dev Biol 2009, 53:1549-1556

31. Watson AJ, Chu S, Sieck L, Gerasimenko O, Bullen T, Campbell F, McKenna M, Rose T, Montrose MH: Epithelial barrier function in vivo is sustained despite gaps in epithelial layers. Gastroenterology 2005, 129:902-912

32. Bullen TF, Forrest S, Campbell F, Dodson AR, Hershman MJ, Pritchard DM, Turner JR, Montrose MH, Watson AJ: Characterization of epithelial cell shedding from human small intestine. Lab Invest 2006, 86:1052-1063

33. Bement WM, Forscher P, Mooseker MS: A novel cytoskeletal structure involved in purse string wound closure and cell polarity maintenance. J Cell Biol 1993, 121:565-578

34. Erjefält JS, Sundler F, Persson CG: Epithelial barrier formation by airway basal cells. Thorax 1997, 52:213-217

35. Pack RJ, Al-Ugaily LH, Morris G, Widdicombe JG: The distribution and structure of cells in the tracheal epithelium of the mouse. Cell Tissue Res 1980, 208:65-84

36. Terranova VP, Rohrbach DH, Martin GR: Role of laminin in the attachment of PAM 212 (epithelial) cells to basement membrane collagen. Cell 1980, 22:719-726

37. Boudreau N, Werb Z, Bissell MJ: Suppression of apoptosis by basement membrane requires three-dimensional tissue organization and withdrawal from the cell cycle. Proc Natl Acad Sci U S A 1996, 93 : 3509-3513

38. Orzekowsky-Schroeder R, Klinger A, Freidank S, Linz N, Eckert S, Hüttmann G, Gebert A, Vogel A: Probing the immune and healing response of murine intestinal mucosa by time-lapse 2-photon microscopy of laser-induced lesions with real-time dosimetry. Biomed Opt Express 2014, 5:3521-3540 